\title{
G0-WISHART DISTRIBUTION BASED CLASSIFICATION FROM POLARIMETRIC SAR IMAGES
}

\author{
G. C. HU*, Q. H. ZHAO \\ Institute for Remote Sencing Science and Application, School of Geomatics, Liaoning Technical University, Fuxin, \\ Liaoning,123000, China - (cmp371284, zqhlby)@163.com
}

Commission I, WG I/3

KEY WORDS: G0-Wishart distribution, Classification, Polarimetric SAR Images, G0-Wishart distance, $H / A / \alpha$ decomposition

\begin{abstract}
:
Enormous scientific and technical developments have been carried out to further improve the remote sensing for decades, particularly Polarimetric Synthetic Aperture Radar(PolSAR) technique, so classification method based on PolSAR images has getted much more attention from scholars and related department around the world. The multilook polarmetric G0-Wishart model is a more flexible model which describe homogeneous, heterogeneous and extremely heterogeneous regions in the image. Moreover, the polarmetric G0-Wishart distribution dose not include the modified Bessel function of the second kind. It is a kind of simple statistical distribution model with less parameter. To prove its feasibility, a process of classification has been tested with the full-polarized Synthetic Aperture Radar (SAR) image by the method. First, apply multilook polarimetric SAR data process and speckle filter to reduce speckle influence for classification result. Initially classify the image into sixteen classes by $H / A / \alpha$ decomposition. Using the ICM algorithm to classify feature based on the G0-Wshart distance. Qualitative and quantitative results show that the proposed method can classify polaimetric SAR data effectively and efficiently.
\end{abstract}

\section{INTRODUCTION}

In the interpreting and analyzing filed of Polarimetric Synthetic Aperture Radar (PolSAR) data, feature classification study plays a key vital role(Zhang, 2015). Speckle noise is a major issue for the task of feature classification by the SAR images (Zhao, 2016). Instead of combating the speckle noise, we designing a intelligent algorithms for land-use, ground cover classification in SAR images based on the statistical properties of speckle(Lee, 2009). It is a fact that the polarimetric covariance matrix obeys the complex Wishart distribution (Papoulis, 2002; Lee, 1994; ). Based on it, Lee proposed a classification algorithm which combining $\mathrm{H} / \mathrm{A} / \alpha$ decomposition with maximum likelihood classifier(Lee, 1999; Pottier, 2000). The complex Wishart distribution agrees reasonably well for measurements over homogeneous backscattering media, but it could not describe the heterogeneous regions in SAR images. For the heterogeneous regions, some new statistical distributions, such as, the $\mathrm{K}$ distribution, have been found to be useful in modeling the polarmetric data(Jakeman,1980; Lee, 1994). Frery, proposed the Polarimetric covariance matrix obeys the G0 polarmetric distribution. Furthermore, the distribution can not only describing well the data which the $\mathrm{K}$ distribution explains, but also explaining extremely heterogeneous regions. Frery, 2006 adopts the G0 distribution to feature classification of multilook fully polarimetric SAR data by ICM. The G0 distribution provides a good and tractable description of extremely heterogeneous areas in PolSAR imagery, it is a special form of $\mathrm{G}$ model(Freitas , 2005; Gambini J, 2008.).

In order to describe homogeneous, heterogeneous and extremely heterogeneous regions in the multilook fully polarimetric SAR image effectively, the paper presents a distribution combining G0 distribution and Wishart distribution. To prove its feasibility, a process of classification has been tested with the full-polarized Synthetic Aperture Radar (SAR) image by the method. Using
NASA/JPL AIRSAR and UAVSAR, L-Band, multi-look fully polarimetric complex images. First of all, to reduce speckle influence for classification result and preserve image resolution, while the original PolSAR image does not have a large enough Equivalent Number of Looks(ENL), we choose multilook polarimetric SAR data process by averaging several independent 1-look coherent matrices and speckle filter with a small window(Lopez-Martinez, 2005). Apply zones in the $\mathrm{H} / \mathrm{A} / \alpha$ plane to initially classify the image into sixteen classes. Then, Compute the initial class center $\mathrm{V}$ for pixels located in each class and the G0-Wishart distance between each pixel and class center. Assign the pixel to the class with the minimum distance measure. Finally, By thresholding the number of pixels switching classes and a prespecified number of iterations, the iterative procedure would be terminated ( $\mathrm{Du}, 1996)$. The experimental results indicate that the proposed classifier can classify features precisely.

The reminder of this paper is organized as follows. Section 2 gives the $H / A / \alpha$ decomposition is introduced to initially classify the image. Then a classification model based on the G0-Wisahrt distance which is used as a measure to reassign the pixel to the class. We then in section 3 detail and discuss the result of fully PolSAR images. Finally, conclusion is presented in Section 4.

\section{MODELS FOR POLARIMETRIC DATA}

This Section provides a details discussion of the proposed classification algorithm for fully PolSAR data, which combines the $H / A / \alpha$ decomposition, G0-Wishart distribution, and Iterated Conditional Modes Algorithm (ICM).

\section{$2.1 \quad H / A / \alpha$ decomposition}

$H / A / \alpha$ decomposition is a typical target decomposition method Fully polarimetric SAR image, represented by a polarimetric coherence matrix $\boldsymbol{T}$ of pixel, is defined on the image domain $\boldsymbol{D}$,

\footnotetext{
* Corresponding author: Guangchen Hu, E-Mail: cmp371284@163.com
} 
that is, $\boldsymbol{T}=\left\{T_{d}, d=1, \ldots, m\right\}$, where $d \in \boldsymbol{D}$ is a pixel location on , $\mathrm{m}$ is the total number of $\boldsymbol{D}, T_{d}$ is a random variable defined on $d$.

Polarimetric coherence matrix $T_{d}$ of pixel could be decomposed three independent targets. This decomposition can be written as follows:

$$
T_{d}=\sum_{i=1}^{3} \lambda_{i} u_{i} \cdot u_{i}^{\mathrm{T} *}=k_{i} \cdot k_{i}^{\mathrm{T} *}
$$

where $\quad T_{d}=$ the complex coherence matrix

$\lambda_{i}=$ the eigenvalues of $T_{d}$

$u_{i}=$ the unit orthogonal eigenvectors

$k_{i}=$ the target vector

$\mathrm{T}=$ transposition

$*=$ the conjugate

$i=1,2,3$

A parameterization of the eigenvectors of the coherency matrix given by

$$
u_{i}=\left[\begin{array}{lll}
\cos \alpha_{i} & \sin \alpha_{i} \cos \beta_{i} \mathrm{e}^{j \delta_{i}} & \sin \alpha_{i} \sin \beta_{i} \mathrm{e}^{j \gamma_{i}}
\end{array}\right]^{\mathrm{T}}
$$

where

$$
\begin{aligned}
& \alpha_{i}=\text { the scattering angel } \\
& \beta_{i}=\text { the orientation of the radar line of sight } \\
& \delta_{i}, \gamma_{i}=\text { the phase angles of the target material }
\end{aligned}
$$

Three polarimetric parameters were calculated to describe the polarimetric coherence matrix by the eigenvalue analysis. The probability of $\lambda_{i}$ was given by

$$
P_{\mathrm{i}}=\lambda_{\mathrm{i}} / \sum_{i=1}^{3} \lambda_{i}
$$

The polarimetric entropy $\mathrm{H}$ is defined to describe the degree of the statistical disorder. It can be expressed as

$$
H=-\sum_{i=1}^{3} P_{i} \log _{3}\left(P_{i}\right)
$$

Relative to polarimetric entropy, the anisotropy A is defined to describe the second and the third eigenvalues as a complementary parameter.

$$
A=\frac{\lambda_{2}-\lambda_{3}}{\lambda_{2}+\lambda_{3}}
$$

The scattering angel is defined to identify the dominant scattering mechanism. It can calculated as

$$
\alpha=\sum_{i=1}^{3} p_{i} \alpha_{i}
$$

According to the available zones of 3-D $H / A / \alpha$ plane which indicate the characteristic of classes of different scattering behavior, initially classify the image into sixteen classes.

\subsection{G0-Wishart distribution}

In order to obtains a distribution to interpret all kind of roughness regions, the paper combining the G0 distribution and Wishart distribution. For multilook polarmetric SAR data, it is necessary to average several independent 1-look covariance matrices. The n-look covariance matrix could be expressed as

$$
C_{d}=\frac{1}{n} \sum_{k=1}^{n} e(k) e(k)^{* \mathrm{~T}}
$$

where

$n=$ the number of look used to generate the image

$$
e(k)=\text { the } k \text { th 1-look sample }
$$

Let $Z_{d}=n C_{d}$. The n-looks polarmetric complex covariance matrix obeys the multivariate G0-Wishart distribution with probability distribution function(PDF) given by

$$
\begin{gathered}
f\left(Z_{d}\right)=\frac{n^{q n} Z_{d}{ }^{n-q} \Gamma(q n-\alpha) \exp \left[-\operatorname{Tr}\left(V^{-1} Z_{d}\right)\right]}{R(n, q)|V|^{n+1} \Gamma(-\alpha)(-\alpha-1)^{\alpha}} \\
\cdot\left(n \operatorname{Tr}\left(V^{-1} Z_{d}\right)-\alpha-1\right)^{\alpha-q n}, \alpha<-1
\end{gathered}
$$

where $\quad Z_{d}=$ the n-looks complex covariance matrix

$q=$ the number of channel

$\alpha=$ the roughness parameter

$V=$ the class center

$\mathrm{R}(n, q)=\pi^{q(q-1) / 2} \Gamma(n) \cdots \Gamma(n-q+1)$

$\Gamma(\cdot)=$ Gamma function

$\operatorname{Tr}(\cdot)=$ the trace of matrix

$\mathrm{R}(n, q)=$ the number of channel

$|\cdot|=$ the determinant of matrix

This multivariate distribution is a kind of simple statistical distribution model with less parameter which dose not include the modified Bessel function of the second kind. The parameter $q$ is number of channel is related to the chosen image. In this paper, the data is monostatic polarimetric SAR on a reciprocal medium, so the number of channel $q$ are 3 . The roughness parameter $\alpha$ of pix is the mean of the parameter of every channel $\alpha_{\mathrm{HH}}, \alpha_{\mathrm{HV}}, \alpha_{\mathrm{VV}}$. Take HH polarimetric SAR image as example, in order to effectively estimate roughness parameter $\alpha_{\mathrm{HH}}$, the first and second moments of the distribution were chosen to parameter estimation by the analogy method. The roughness parameter $\alpha_{\mathrm{HH}}$ is then estimated with

$$
\alpha_{\mathrm{HH}}=\frac{m_{1}^{2}(n+1)}{n m_{2}-m_{1}^{2}(n+1)}
$$

where $\quad m_{1}=$ the first moment

$$
m_{2}=\text { the second moment }
$$

The first and second moments are given by

$$
\begin{aligned}
& m_{1}=E\left(\left|S_{\mathrm{HH}}\right|\right) \\
& m_{2}=E\left(\left|S_{\mathrm{HH}}\right|^{2}\right)
\end{aligned}
$$

where $S_{\mathrm{HH}}=$ the scattering element of $\mathrm{HH}$ polarimetric $\mathrm{E}(\cdot)=$ sample mean of the pix

\subsection{G0-Wishart classifier}

The typical classifiers include a distance measure classifier and Bayes maximum likelihood classification. Relative to the maximum probability density functions for selecting the class, we choose the more simpler and effective classifier which distance measure by taking the natural logarithm of probability density functions and changing its sign. Taking the natural logarithm of Equation (8) and changing its sign, we have

$$
\begin{gathered}
d\left(Z_{d}, \omega_{m}\right)=(n+1) \ln \left|V_{m}\right|-\ln \Gamma(q n-\alpha)+\operatorname{Tr}\left(V_{m}^{-1} Z_{d}\right)+\ln \Gamma(-\alpha) \\
-(\alpha-q n) \ln \left(n \operatorname{Tr}\left(V_{m}^{-1} Z_{d}\right)-\alpha-1\right)+\alpha \ln (-\alpha-1) \\
+\ln R(n, q)-(n-q) \ln \left(Z_{d}\right)-q n \ln n
\end{gathered}
$$


where $\ln (\cdot)=$ the natural logarithm

$V_{m}=$ the class center of the $\omega_{m}$ class

The class center of $m$ class was given by

$$
V_{m}=\frac{\sum_{n=1}^{N_{m}} C_{m n}}{N_{m}}, n=1,2, \cdots N_{m}
$$

where $\quad C_{m n}=$ the covariance matrix of first n pix of $\omega_{m}$ class $N_{m}=$ pixel count of the $\omega_{m}$ class

The last three terms of Equation (11) no relationship with class center $V_{m}$ and do not contribute to the classification, so they would be ignored. Deleting the last three terms of Equation (11), the distance measure for classification of n-look processed polarimetric SAR data becomes

$$
\begin{aligned}
d\left(Z_{d}, \omega_{m}\right)= & (n+1) \ln \left|V_{m}\right|+\alpha \ln (-\alpha-1)-\ln \Gamma(q n-\alpha)+\ln \Gamma(-\alpha) \\
& +n \operatorname{Tr}\left(V_{m}^{-1} Z_{d}\right)-(\alpha-q n) \ln \left(n \operatorname{Tr}\left(V_{m}^{-1} Z_{d}\right)-\alpha-1\right)
\end{aligned}
$$

The distance measure could indicate the degree of between pix and class, if the distance $d\left(Z_{d}, \omega_{m}\right)$ was minimum, the pix is divided into $\omega_{m}$ class.

\subsection{Implementation of G0-Wishart classifier}

G0-Wishart classifier is described in the flowchart of Fig. 1, and the details of the implementation are provided in the following.

1) Multilook process for polarimetric SAR data by averaging several independent 1-look coherent matrices. Using Refined Lee filter to reduce speckle influence for processed coherence matrix.

2) Compute the target coherence matrix $T$, apply $H / A / \alpha$ decomposition. Diagonalize the coherence matrix, and generate the normalized eigenvalues $\lambda_{i}$. Cumpute the entropy $\mathrm{H}$, polarimetric anisotropy A, scattering angle $\alpha$.

3) Apply zones in the $H / A / \alpha$ plane to initially classify the image into sixteen classes.

4) Convert the polarimetric coherency matrices into covariance matrices.

5) Compute the initial class center $V$ for pixels located in each class and the G0-Wishart distance between each pixel and class center.

6) Assign the pixel to the class with the minimum distance measure.

7) According to G0-Wishart distance between classes which indicate the degree of separation between two clusters, the similar class would be merge.

8) By thresholding the relevant parameter, for instance, G0Wishart dispersion within class, the number of pixels switching classes and a prespecified number of iterations, the iterative procedure would be terminated.

\section{CLASSIFICATION OF FULLY POLARIMETRIC SAR IMAGES}

To prove its feasibility, the G0-Wishart classifier is tested with two full-polarized Synthetic Aperture Radar (SAR) images acquired by Jet Propulsion Laboratory(JPL), including a small, simple region and a complex, big region. The first is an agricultural area from an airborne NASA/JPL AIRSAR, L-band, quad-Pol SAR flight over Flevoland Nederland in 1989. In addition, the image are also used to quantitatively evaluate the propose algorithm. And the other is from an UAVSAR, L-band, quad-Pol ASAR image over California, America in 2011.

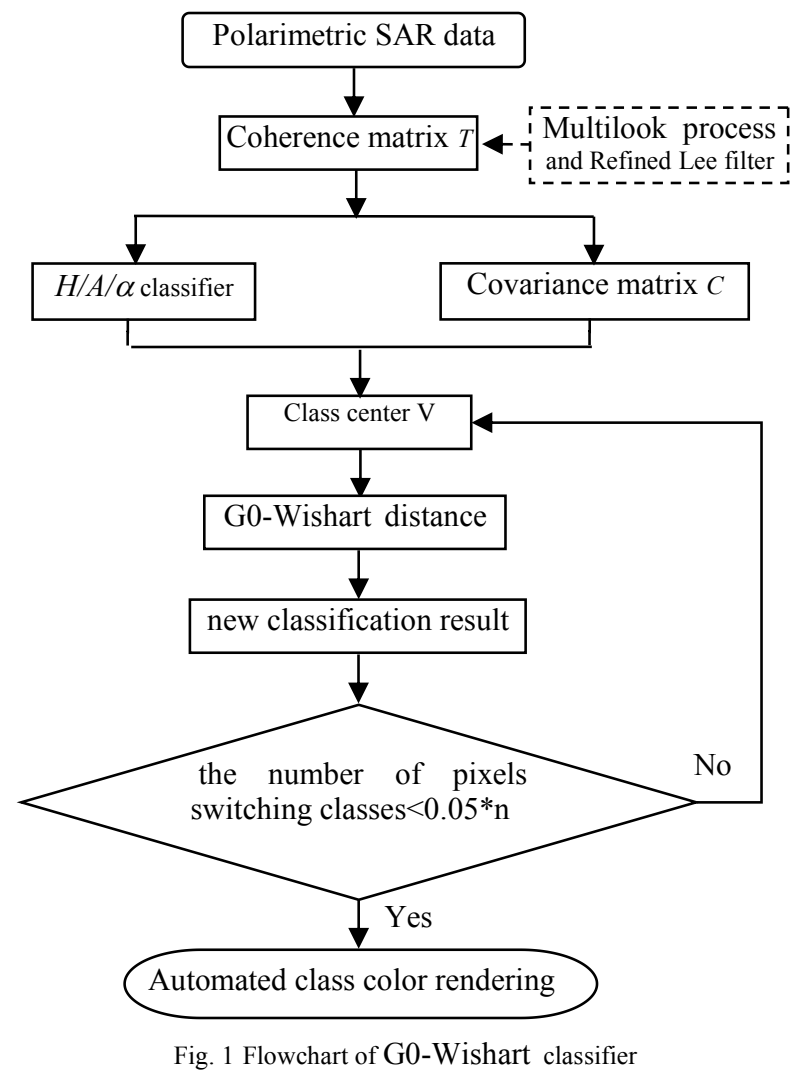

3.1 Result and evaluation of Small area

To evaluate the performance of proposed approach conveniently, the experiment chose an agricultural area from an airborne NASA/JPL AIRSAR, L-band, quad-pol SAR flight over Flevoland Nederland in 1989 as data source(Churchill, 1994). The pixel size is $6.6 \mathrm{~m}$ in the slant range direction and $12.10 \mathrm{~m}$ in the azimuth direction. The incidence angles are 19.78 at near range and 44.18 at far range. The pseudo color image composed by Pauli decomposition is shown in Fig. 2(a). It is a four complex $190 \times 270$ pixels image. The Fig. 2 (b) is the original ground truth map. It identified a total of 7 crop classes, include bare soil, wheat, beet, potato, barley, lucerne, pea. The color coded class label is given in Figure 2(c).

To demonstrate the efficiency of the proposed approch, the result of the proposed method and the contrast experiment is shown in Fig. 3. Using $H / A / \alpha$ decomposition and G0-Wishart classifier, the classification results are shown in Fig. 3(a). To qualitatively evaluate the proposed approach, the result of $H / A / \alpha$-Wishart classifier and the G0 classifier are presented, see Fig. 3(b)-(c). Visually, relative to $H / A / \alpha$-Wishart classifier, the G0 classifier and G0-Wishart classifier classify the all crop classes precisely.

To perform accuracy estimates quantitatively, apply the average accuracy to evaluate classification result. Table 1 lists the classification accuracy of three algorithms. From Table 1, it can be seen that all the accuracy of G0-Wishart classifier indexes are least $90 \%$. It can be illustrate that the proposed approach is feasible and effective. Because of the tested image is simple, the advantage of proposed approach might be not obvious than G0 classifier. 


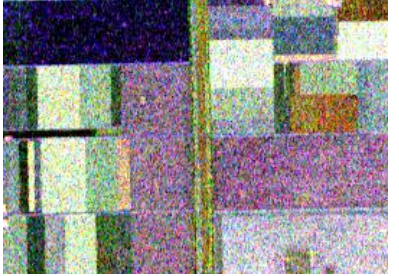

(a)

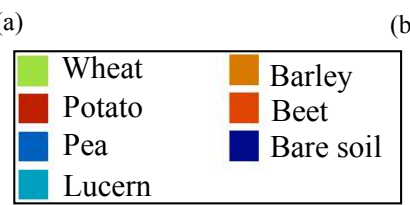

(c)

Fig. 2 (a) Pauli RGB image, (b) Original ground truth map, (c) Class label

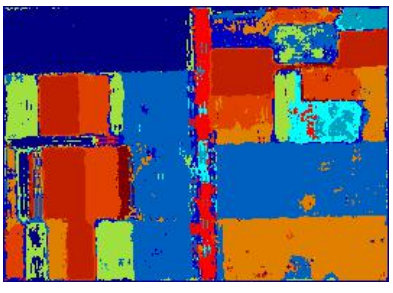

(a)

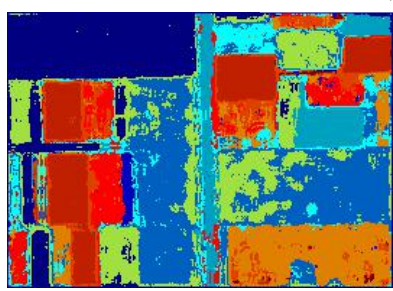

(b)

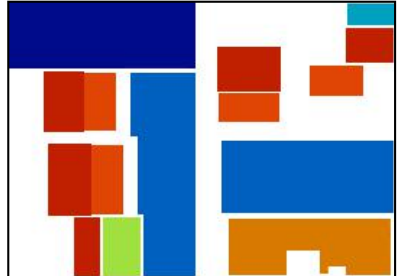

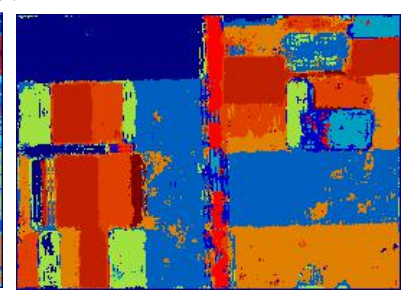

(c)
Fig. 3 (a) $H / A / \alpha$-G0-Wishart classifier, (b) $H / A / \alpha$-Wishart classifier, (c) $H / A / \alpha-\mathrm{G} 0$ classifier

\begin{tabular}{|c|c|c|c|}
\hline Class & $\begin{array}{l}\text { G0-Wishart } \\
\text { classifier } \%\end{array}$ & $\begin{array}{c}\mathrm{G} 0 \\
\text { classifier/\% }\end{array}$ & $\begin{array}{c}\text { Wishart } \\
\text { classifier } \%\end{array}$ \\
\hline Bare soil & 98.87 & 99.82 & 99.20 \\
\hline potato & 93.85 & 95.10 & 85.01 \\
\hline beet & 93.24 & 91.92 & 72.45 \\
\hline barley & 97.81 & 97.78 & 86.59 \\
\hline wheat & 90.24 & 88.93 & 85.46 \\
\hline pea & 95.16 & 91.47 & 73.04 \\
\hline lucerne & 90.51 & 92.72 & 85.66 \\
\hline $\begin{array}{c}\text { average } \\
\text { accuracy } / \%\end{array}$ & 95.49 & 94.19 & 82.09 \\
\hline
\end{tabular}

Table 1 . The classification accuracy

\subsection{Result of complex area}

To perform the capacity of proposed approach describe complex area further, a complex and big image is tested by the proposed approach. Fig. 4(a) shows the Pauli RGB image with size of 829 $\times 829$ pixels. The pixel size is $7.2 \mathrm{~m}$ in the slant range direction and $5 \mathrm{~m}$ in the azimuth direction. See the Fig. 4(a), the top left corner(white area) is residential areas, the bottom is mountain, the other area(image right center) is agricultural area. Fig. 4(b)(d) show the results of G0-Wishart classifier, Wishart classifier, and G0 classifier, respectively. It can be seen that the G0Wishart classifier can not only classify the agricultural area, but also segment the residential areas precisely. By contrast, in the result of Wishart classifier, the residential areas has a lot of noise. In the result of G0 classifier, the residential areas was confused with some crop classes. So it can be demonstrate that

the proposed approach is suitable to classify the image of various terrain

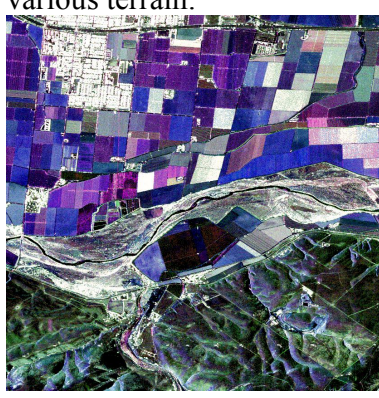

(a)

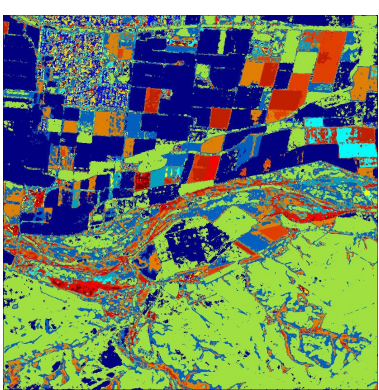
(c) $H / A / \alpha$-Wishart classifier (d) $H / A / \alpha$-G0 classifier

\section{CONCLUSION}

In this paper, a unsupervised classifier for full-Pol SAR data has been developed, which is based on $H / A / \alpha$ decomposition and G0-Wishart distribution. Here, relative to the common distribution, such as Wishart distribution and G0 distribution, the multilook polarmetric G0-Wishart model is a more flexible model which describe homogeneous, heterogeneous and extremely heterogeneous regions in the image. The experimental results indicate that the proposed classifier can not only classify the various terrain precisely but also reduce the speckle accurately.

\section{ACKNOWLEDGEMENTS}

This work was supported by the National Natural Science Foundation of China (No.41271435; No.41301479) and the Natural Science Foundation of Liaoning Province, China (No. 2015020090)

\section{REFERENCES}

Churchill, ... (c) 994. The MAESTRO 1 European airborne polarimetric synthetic aperture radar campaign. International journal of remote sensing, 15(14), pp. 2707-2717.

Du, L., 1996. Fuzzy classification of earth terrain covers using complex polarimetric SAR data. International Journal of Remote Sensing,17(4), pp. 809-826.

Freitas, C. C., 2005. The polarimetric G distribution for SAR data analysis. Environmetrics, 16(1), pp. 13-31.

Frery, A., 2006. Multifrequency Full Polarimetric SAR Classification with Multiple Sources of Statistical Evidence[C]// IEEE International Conference on Geoscience and Remote Sensing Symposium. IEEE, pp. 4195-4197. 
Gambini, J., 2008. Accuracy of edge detection methods with local information in speckled imagery. Statistics and Computing, 18(1), pp. 15-26.

Lee, J. S., 1994. Classification of multi-look polarimetric SAR data based on complex Wishart distribution[J]. International Journal of Remote Sensing, 15(11), pp. 2299-2311.

Jakeman, E. 1980. On the statistics of K-distributed noise. Journal of Physics A: Mathematical and General, 13(1), pp. 31 .

Lee, J. S., 1994. K-distribution for multi-look processed polarimetric SAR imagery. In International Geoscience and Remote Sensing Symposium. IEEE, Vol. 4, pp. 2179-2181.

Lee, J. S., 2009. Polarimetric Radar Imaging: From Basics to Applications[M].

Lee, J. S., 1999. Unsupervised classification using polarimetric decomposition and the complex Wishart classifier. IEEE Transactions on Geoscience and Remote Sensing, 37(5), pp. 2249-2258.
Lopez-Martinez, C., 2005. Statistical assessment of eigenvectorbased target decomposition theorems in radar polarimetry. IEEE Transactions on Geoscience and Remote Sensing, 43(9), pp. 2058-2074.

Papoulis, A., 2002. Probability, random variables, and stochastic processes. Tata McGraw-Hill Education.

Pottier, E., 2000. Unsupervised classification scheme of POLSAR images based on the complex Wishart distribution and the «H/A/alpha» polarimetric decomposition theorem (polarimetric SAR). In EUSAR 2000 , pp. 265-268.

Zhang, L., 2015. Fully polarimetric SAR image classification via sparse representation and polarimetric features.IEEE Journal of Selected Topics in Applied Earth Observations and Remote Sensing, 8(8), pp. 3923-3932.

Zhao, Q. H., 2016. SAR Image Segmentation with Unknown Number of Classes Combined Voronoi Tessellation and Rjmcmc Algorithm. III-7. pp. 119-124. 